\title{
In-Situ Metabolomic Analysis of Setaria viridis Roots Colonized by Beneficial Endophytic Bacteria
}

\author{
Beverly J. Agtuca, ${ }^{1}$ Sylwia A. Stopka, ${ }^{2}$ Thalita R. Tuleski, ${ }^{1,3}$ Fernanda P. do Amaral, ${ }^{1}$ Sterling Evans, ${ }^{1}$ \\ Yang Liu, ${ }^{4}$ Dong Xu, ${ }^{4}$ Rose Adele Monteiro, ${ }^{3}$ David W. Koppenaal, ${ }^{5}$ Ljiljana Paša-Tolić, ${ }^{5}$ \\ Christopher R. Anderton, ${ }^{5}$ Akos Vertes, ${ }^{2}$ and Gary Stacey ${ }^{1,+}$ \\ ${ }^{1}$ Divisions of Plant Sciences and Biochemistry, Christopher S. Bond Life Sciences Center, University of Missouri, Columbia, MO \\ 65211, U.S.A. \\ 2 Department of Chemistry, The George Washington University, Washington, DC 20052, U.S.A. \\ ${ }^{3}$ Department of Biochemistry and Molecular Biology, Federal University of Paraná, CP 19046, 81.531-990 Curitiba, PR, Brazil \\ ${ }^{4}$ Department of Electrical Engineering and Computer Science, Informatics Institute and Christopher S. Bond Life Sciences \\ Center, University of Missouri Columbia \\ ${ }^{5}$ Environmental Molecular Sciences Laboratory, Earth and Biological Sciences Directorate, Pacific Northwest National \\ Laboratory, 902 Battelle Boulevard, Richland, WA 99354, U.S.A.
}

Accepted 17 September 2019.

\begin{abstract}
Over the past decades, crop yields have risen in parallel with increasing use of fossil fuel-derived nitrogen $(\mathrm{N})$ fertilizers but with concomitant negative impacts on climate and water resources. There is a need for more sustainable agricultural practices, and biological nitrogen fixation (BNF) could be part of the solution. A variety of nitrogen-fixing, epiphytic, and endophytic plant growth-promoting bacteria (PGPB) are known to stimulate plant growth. However, compared with the rhizobium-legume symbiosis, little mechanistic information is available as to how PGPB affect plant metabolism. Therefore, we investigated the metabolic changes in roots of the model grass species Setaria viridis upon endophytic colonization by Herbaspirillum seropedicae $\operatorname{SmR1}\left(\mathrm{fix}^{+}\right.$) or a fix ${ }^{-}$mutant strain (SmR54) compared with uninoculated roots. Endophytic colonization of the root is highly localized and, hence, analysis of whole-root segments dilutes the metabolic signature of those few cells impacted by the bacteria. Therefore, we utilized insitu laser ablation electrospray ionization mass spectrometry to sample only those root segments at or adjacent to the sites of bacterial colonization. Metabolites involved in purine, zeatin,
\end{abstract}

Current address for Beverly J. Agtuca: Department of Biology and Geosciences, Adams State University, Alamosa, CO 81101, U.S.A.

${ }^{\dagger}$ Corresponding author: G. Stacey; staceyg @ missouri.edu

Funding: This research was supported by the United States Department of Energy (DOE), Office of Biological and Environmental Research (OBER) under award number DE-SC0013978 and National Science Foundation (NSF) Plant Genome Program under award number IOS-1734145. B. J. Agtuca thanks the U.S. DOE Mickey Leland Energy Fellowship and the University of Missouri College of Agriculture, Food and Natural Resources (CAFNR) and Office of Graduate Studies for the George Washington Carver Fellowship and Gus T. Ridgel Fellowship. S. Evans was supported by the University of Missouri Freshman Research in Plant Sciences (FRIPS) program.

*The $\boldsymbol{e}$-Xtra logo stands for "electronic extra" and indicates that five supplementary figures and four supplementary tables are published online.

The author(s) declare no conflict of interest.

○ 2020 The American Phytopathological Society and riboflavin pathways were significantly more abundant in inoculated plants, while metabolites indicative of nitrogen, starch, and sucrose metabolism were reduced in roots inoculated with the fix ${ }^{-}$strain or uninoculated, presumably due to $\mathbf{N}$ limitation. Interestingly, compounds, involved in indolealkaloid biosynthesis were more abundant in the roots colonized by the fix ${ }^{-}$strain, perhaps reflecting a plant defense response.

Keywords: associative bacteria, Herbaspirillum seropedicae, laser ablation electrospray ionization mass spectrometry, metabolites, nifA, nitrogen fixation, PGPB, plant growth promotion, rhizosphere

Plant development and productivity rely on nutrients that are naturally available in the soil. However, in many situations, specific nutrients necessary for plant growth are present in low abundance or may not be available in a form that can be readily absorbed by the roots. For instance, nitrogen is a critical macronutrient for plant growth and is commonly a limiting nutrient in many environments. $\mathrm{N}$ is also the most energy expensive for plants to uptake (Galloway et al. 2004, 2008). Crop production requires large amounts of $\mathrm{N}$ fertilizer for maximum yield, especially for cereals, such as maize and rice (Smil 2001; Godfray et al. 2010; Tilman et al. 2011). However, actual utilization of applied $\mathrm{N}$ fertilizer has an efficiency of $50 \%$ or less (Edmonds et al. 2013; Raun and Johnson 1999). Thus, improvement in nitrogen use efficiency is needed and is essential for sustainable and eco-friendly agriculture.

The overuse of nitrogen fertilizer leads to detrimental soil and environmental consequences. Hence, a major challenge for sustainable agricultural production is how to deliver nitrogen to the plant to maintain high yield while negating the negative consequences of adding nitrogen fertilizer (Dobermann 2007; Sutton et al. 2011; Westhoff 2009). In this context, the use of biological nitrogen fixation (BNF) has often been proposed as one possible solution to at least reduce if not to eliminate the need for heavy $\mathrm{N}$ fertilization of nonlegume crops (Franche et al. 2009; Lugtenberg and Kamilova 2009). However, in most situations, the contribution of BNF to growth induced by plant growth-promoting bacteria (PGPB) remains unclear or 
undefined (Franche et al. 2009; Lugtenberg and Kamilova 2009).

A variety of BNF bacteria are commonly present in the plant rhizosphere that can establish close associations with roots, colonizing the roots either epiphytically or endophytically. Indeed, PGPB can reach high numbers (e.g., $10^{8}$ per gram) in roots without inducing a noticeable plant defense response (do Amaral et al. 2017; Faoro et al. 2017; Reinhold-Hurek and Hurek 1998, 2011). Previous studies showed that PGPB commonly impact root architecture and plant health, attributing these effects to such things as BNF, enhancing stress tolerance, production of phytohormones, enhancing nutrient acquisition, and protection against pathogens and pests (Pankievicz et al. 2015; Pérez-Montaño et al. 2014). Nevertheless, definitive evidence that defines the specific mechanism of PGPBmediated plant growth promotion remains lacking. In similar plant-microbe interactions (e.g., legume symbiosis), the use of one or both bacterial and plant mutants have been particularly useful in defining molecular mechanism. For example, our laboratory recently demonstrated that disruption of the genes for biosynthesis and utilization of poly-3-hydroxybutyrate (PHB) in the endophytic bacterium Herbaspirillum seropedicae directly affected its ability to promote the growth of Setaria viridis (Alves et al. 2019). PHB is a type of polyhdroxyalkanoate (PHA) polymer, produced as a carbon storage compound by a variety of bacterial species. The PHB cycle provides carbon skeletons to synthetic and energetic metabolism as well as providing reducing power for nitrogen fixation (Lodwig et al. 2005). A few other studies have identified other genes essential for plant growth promotion in other PGPB (Krause et al. 2006; Sarkar and Reinhold-Hurek 2014; Sessitsch et al. 2012; Shidore et al. 2012). However, while much remains to be done from both the bacterial and plant side, data are particularly missing regarding the molecular response of the plant host to PGPB association.

In order to define the plant response to PGPB, researchers are applying the full repertoire of modern technologies, including transcriptomics, proteomics, and metabolomics. While the detection of specific transcripts and proteins provides evidence of the potential for a function or pathway to be active, it is only metabolomic analysis that provides definitive evidence that, indeed, specific metabolism is occurring. There are, for example, specific studies in which metabolomic analysis was nicely integrated into efforts of crop improvement (Kumar et al. 2017; Zivy et al. 2015). Studies in oats, for instance, identified specific metabolic pathways involved with drought tolerance (Sánchez-Martín et al. 2015) and similar efforts identified metabolite-phenotype associations for selecting drought-tolerant ecotypes of Brachypodium spp. (Fisher et al. 2016). However, few studies have used metabolomics to investigate PGPB-plant interactions. One example is BrusamarelloSantos et al. (2017), who profiled the metabolite distribution of two inbred maize lines upon inoculation with the diazotrophic PGPB Azospirillum brasilense and Herbaspirillum seropedicae.

A general limitation with most published metabolomic studies is that they rely on bulk analysis from whole tissues, when it is clear, for example, that PGPB colonization of plant roots is highly localized. A diversity of technologies and methodologies are currently available for such a large-scale metabolomic analysis, including nuclear magnetic resonance (NMR) spectrometry, gas chromatography-mass spectrometry (GCMS), liquid chromatography (LC)-MS, capillary electrophoresis (CE)-MS, and tandem mass spectrometry (MS/MS) (Gemperline et al. 2016; Mhlongo et al. 2018; Tenenboim and Brotman 2016). However, in addition to generally requiring a significant amount of starting plant tissue, these methods are also usually low throughput and do not support in-situ analysis. Therefore, especially when examining localized areas of PGPB colonization of roots, technologies that allow in-situ metabolic profiling and imaging of biological tissues via a high-throughput approach have clear advantages.

Metabolomic methods that can be performed in situ and spatially explicit, commonly suffer from required in-depth and challenging sample preparation procedures. For example, matrix-assisted laser desorption-ionization MS is a method capable of routine, relatively high lateral resolution molecular imaging $(10 \mu \mathrm{m})$ but requires extensive sample preparation, including spraying a weak organic acid onto the sample, that assists in facilitating desorption and ionization of molecules (Gemperline et al. 2016; Veličković et al. 2018). In contrast, ambient ionization MS-based approaches, such as laserablation electrospray ionization (LAESI), involve minimal sampling methods, while acquiring spatial information of metabolites in biological tissues in their native conditions (Müller et al. 2011; Nemes and Vertes 2007; Nemes et al. 2009; Stopka et al. 2019). Previously, we demonstrated the utility of using LAESI-MS with ion mobility separation to explore the spatial distribution of metabolites in soybean root tissues and nodules infected with BNF rhizobia (Stopka et al. 2017). We also demonstrated that LAESI-MS is useful in identifying the metabolite changes associated with the use of plant and bacterial mutants defective in the soybean-rhizobia symbiosis (N. Aguiar, L. Medici, F. Olivares, L. Dobbss, A. Torres-Netto, S. Silva, E. Novotny, and L. Canellas, unpublished).

In this study, we demonstrate the utility of LAESI-MS to profile the metabolites associated with localized regions of Setaria viridis roots colonized by an endophytic bacterium, Herbaspirillum seropedicae SmR1, and, for comparison, a corresponding mutant (SmR54) lacking functional nitrogenase activity (Roncato-Maccari et al. 2003). SmR54 is a nifA mutant strain, in which NifA functions as a transcriptional activator for nif gene expression (Sarkar and Reinhold-Hurek 2014). This work builds on our previous work, in which, using nitrogen-13 tracer studies, we showed that $S$. viridis, at least under defined laboratory conditions, obtained a significant amount of its $\mathrm{N}$ needs from PGPB-mediated BNF (Pankievicz et al. 2015).

\section{RESULTS}

Our past work revealed that coinoculation of $H$. seropedicae SmR1 and Azosprillum brasilense FP2 resulted in significant growth promotion of $S$. viridis with measurable incorporation of ${ }^{13} \mathrm{~N}_{2}$ and concomitant shifts in the general abundance of leaf amino acid pools (Pankievicz et al. 2015). A more recent study demonstrated that inoculation of $S$. viridis solely with $H$. seropedicae $\mathrm{SmR} 1$ resulted in a significant increase of plant growth within 25 days postinoculation (Alves et al. 2019). Collectively, these studies demonstrate the ability of $H$. seropedicae to stimulate $S$. viridis growth and the potential for significant effects on plant metabolism. Hence, we focused on this interaction in order to better define the plant metabolomic response, especially at the specific sites of bacterial colonization of the root.

\section{Bulk metabolomics of $S$. viridis roots colonized by $\boldsymbol{H}$. seropedicae.}

In order to bolster confidence in our later LAESI-MS analyses, we first used bulk extraction of $S$. viridis plant tissues to sample the metabolome. The experimental samples were derived from 2-week-old Setaria plants inoculated with either H. seropedicae strain, $\mathrm{SmR} 1\left(\mathrm{fix}^{+}\right.$) or $\mathrm{SmR} 54$ (fix ${ }^{-}$), compared with control uninoculated plants (CTRL). The whole plant roots were ground and extracted in methanol, and those 
samples, with 20 replicates in each sample group, were analyzed by LAESI-MS (Supplementary Fig. S1A). Multivariate statistical analysis was performed and showed that all three groups overlapped with no degree of separation, according to the partial least squares discriminant analysis (PLS-DA) (Supplementary Fig. S1B). In total, we detected about 130 spectral features, with none showing significant differences in abundance based on treatment (Supplementary Fig. S1C). We interpret these findings to be the result of highly localized zones of bacterial colonization, hence, diluting out any effects that would be infection-site specific.

\section{Spatial information in specific root segments colonized} by endophytic bacteria.

Instead of bulk analysis, $S$. viridis root segments taken from areas with the highest level of colonization (as shown by green fluorescent protein [GFP]) were selected in order to specifically observe host response. These samples were then analyzed by LAESI-MS. Again, root segments inoculated with H. seropedicae strains SmR1 or SmR54 or uninoculated were compared (Supplementary Fig. S2). Based on GFP expression by the colonizing bacteria, specific root segments were selected, cut, and flash frozen for subsequent LAESI-MS analyses (Fig. 1). As described below, comparable roots were used to quantify the level of bacterial colonization (Supplementary
Fig. S3B), demonstrating that $H$. seropedicae SmR1 or SmR54 colonized Setaria roots to equivalent levels. Measurements of root and shoot biomass of inoculated plants, relative to uninoculated plants, showed significant growth promotion regardless of BNF (Supplementary Fig. S3A), similar to our recently published study (Alves et al. 2019).

Using this approach, six biological replicates in each sample group were examined, and our data showed a clear distinction based on treatment (Fig. 2), in sharp contrast to our bulk analysis (Supplementary Fig. S1). The heat map (Fig. 2A) showed different metabolic patterns in the CTRL and SmR1 roots. In addition, the CTRL and SmR1 root samples contained the most metabolites that differed in abundance compared with the mutant SmR54 samples (Fig. 2A). Additionally, the PLSDA scores plot showed a high spectral similarity within sample groups and a high degree of separation among different sample types (Fig. 2B). Component 1 captured the spectral difference between plants that were inoculated (SmR1 and SmR54) and uninoculated. Component 2 reflected spectral differences between plants based on their ability for BNF, i.e., SmR1 relative to CTRL and SmR54 root segments (Fig. 2B). A total of 305 spectral features were detected, by LAESI-MS, between the CTRL and SmR1 roots. Specifically, the CTRL had 12 significantly upregulated metabolites, whereas SmR1 had 15 upregulated metabolites with biological and statistical cutoffs

\section{Plants grew at 2 weeks after inoculation in greenhouse conditions.}

\section{Setaria A10.1 seedlings were inoculated by PGPB, while the CTRL was uninoculated.}
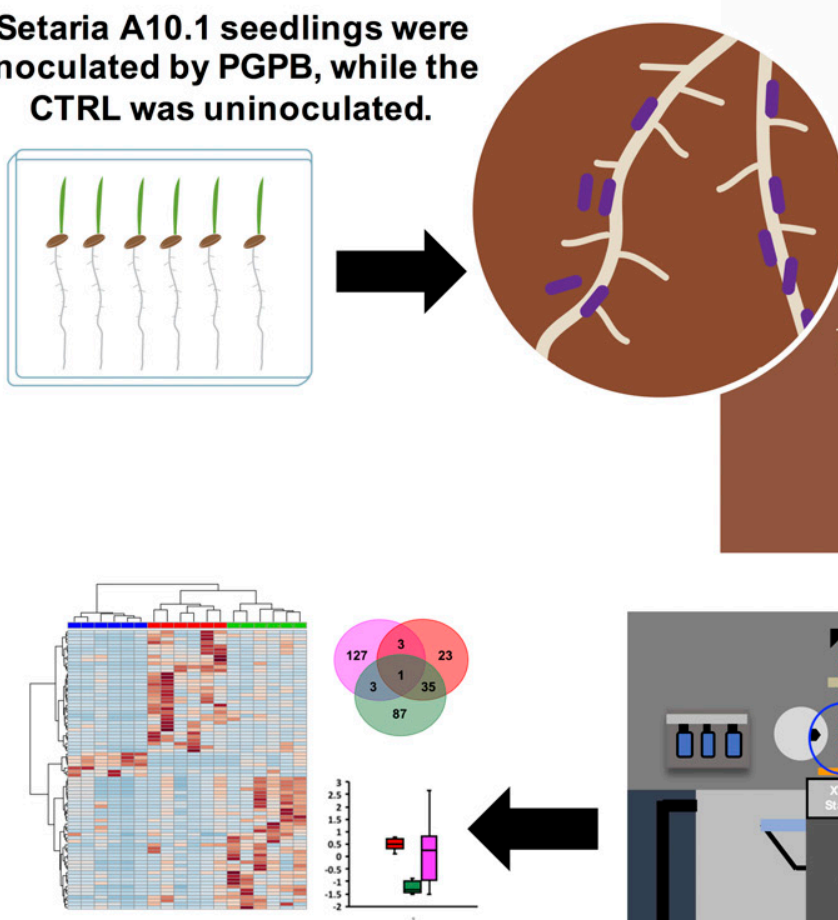

\section{Multivariate statistical analysis}

Roots were harvested and analyzed by the fluorescence microscope.

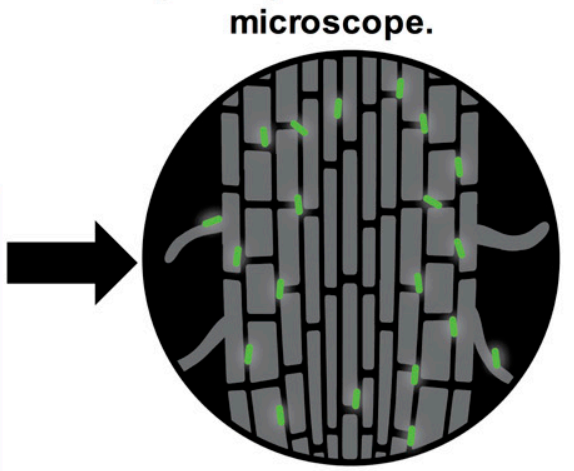

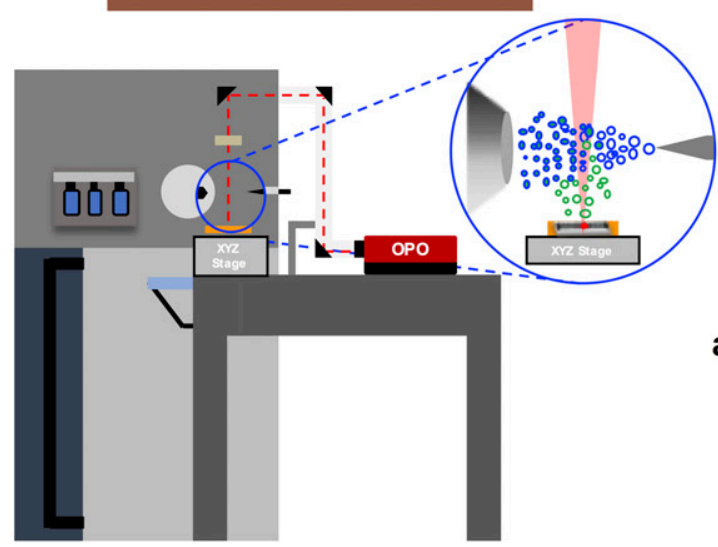

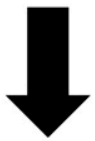

The newly developed

leaves and root segments were analyzed by LAESI-MS.

Fig. 1. Schematic of experimental design. Three-day-old seedlings of Setaria viridis A10.1 were inoculated with either Herbaspirillum seropedicae SmR1 $\left(\right.$ fix $^{+}$) or SmR54 (fix ${ }^{-}$), while the control (CTRL) plants were uninoculated. The plants grew for 2 weeks after inoculation under greenhouse conditions. The roots and leaves were harvested. Roots from plants that were inoculated with SmR1 or SmR54 were analyzed by fluorescence microscopy. The root areas with the highest green fluorescent protein (GFP) expression, indicative of endophytic bacterial colonization, were cut into segments and were used for analyses. The roots from uninoculated control plants were observed by microscopy and screening for GFP to check if there was any bacterial contamination, and, then, comparable root segments were taken for analysis. Finally, the youngest, newly emerged sink leaf, the selected root segments, and the free-living bacterial cultures were analyzed by laser ablation electrospray ionization mass spectrometry, using previously described methods (Stopka et al. (2017)). 
for fold change (FC) $>2$ and a $P$ value of $<0.05$ (Fig. 2C). When comparing the CTRL and SmR54 roots, 135 spectral features were observed, in total. Among these, 27 metabolites were significantly upregulated in the CTRL samples and eight were upregulated in the SmR54 roots. Additionally, a total of 281 peaks were detected between roots inoculated with the two bacterial strains, in which 59 metabolites were notably abundant in the SmR1 roots and four were found upregulated in the SmR54 samples (Fig. 2C). After statistical analyses, there were 36 significantly regulated metabolites with a FC of at least 2 that were identified (Table 1). Figure 2D shows box-andwhisker plots for a few of the metabolites that showed significant changes in abundance. For example, glucose phosphate and hydroxyjasmonic acid glucoside were more abundant in the CTRL than in the inoculated roots. Sequoyitol was significantly increased in the SmR1 roots relative to the SmR54 and CTRL samples, while norajmaline was more abundant in the SmR54 roots than the other samples (Fig. 2D).

The high abundance of glucose phosphate present in the CTRL roots was expected, since it is involved with starch and sucrose metabolism. We assume that carbon metabolism would be affected due to the need for carbon utilization by the colonizing bacteria, especially given the need for energy and reductant to support BNF (Mus et al. 2016). Consistent with our findings, a related study found reduced levels of sugars in
PGPB-inoculated maize grown hydroponically (da Fonseca Breda et al. 2019). Most of the identified flavonoid metabolites were more abundant in plants inoculated with the SmR1 strain, relative to those colonized by the SmR54 mutant (Table 1). This finding is difficult to interpret, since flavonoids play a wide variety of roles in plants. For example, these compounds have been shown to serve in signaling and recognition between symbiotic partners (Balachandar et al. 2006; Hardoim et al. 2008; Shaw et al. 2006; Webster et al. 1998). Flavonoids can also modulate internal plant hormone levels, and they may also be signs of plant defense pathway induction (Falcone Ferreyra et al. 2012; Gough et al. 1997; Marin et al. 2013; Liu and Murray 2016; Subramanian et al. 2007; Tadra-Sfeir et al. 2011). Given the lack of any observable plant defense response and the absence of specific data that flavonoids act as signals to PGPB, we favor the possibility that these flavonoids may be modulating plant metabolism in direct response to colonization. However, at this point, this is merely speculation requiring considerably more work for clarification.

Due to the limitations of our experiments, in some cases, we cannot ascertain definitively whether the metabolites detected in the root segments are of plant or bacterial origin. However, we suggest we are primarily analyzing plant metabolites in these samples, given the overall relative mass of the bacterial and plant tissue being analyzed. To further delineate the origin

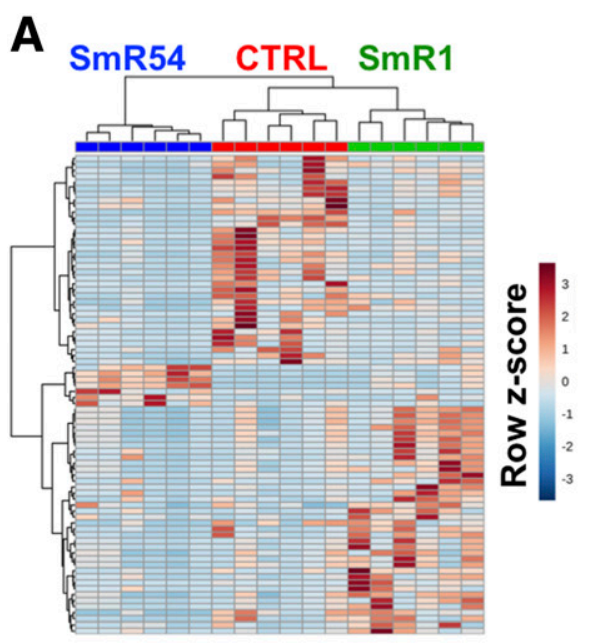

C

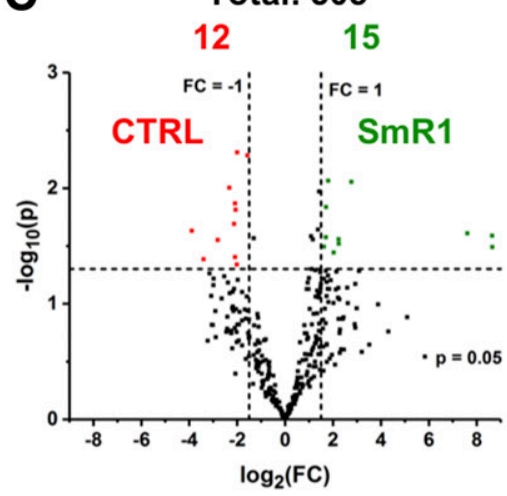

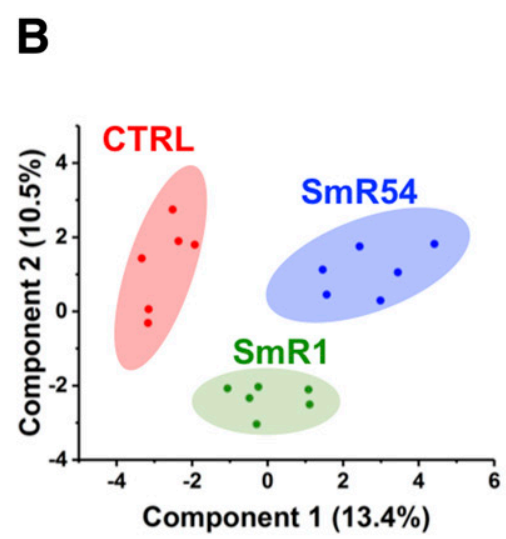
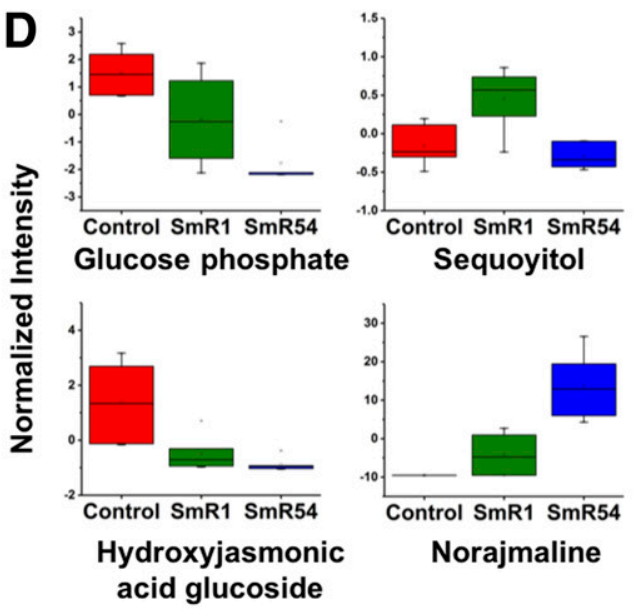

Total: 281

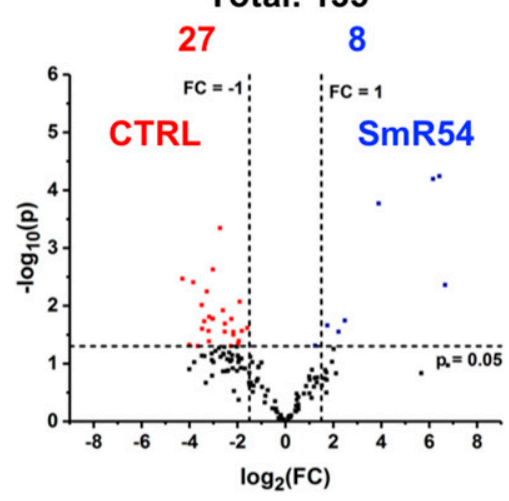

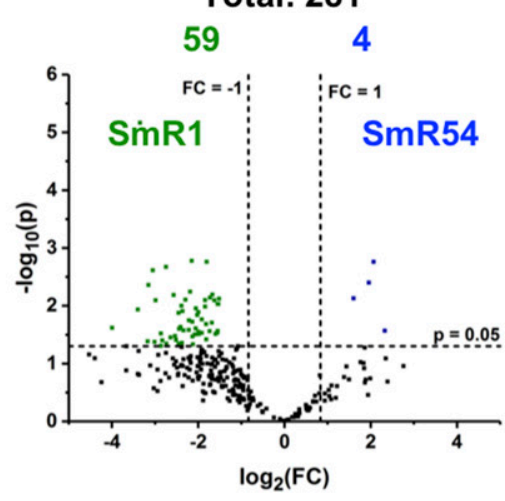

Fig. 2. Comparison of root segments from uninoculated (CTRL) plants (in red) and plants that were inoculated with either SmR 1 (fix ${ }^{+}$) (green) or SmR54 (fix ${ }^{-}$) (blue). A, Heat map of the significant metabolites that were abundant in each sample group. The red row $\mathrm{z}$-score indicates the highest abundance, while the dark blue is the lowest abundance. Each row represents a metabolite, while the column characterizes the biological replicates from each sample group. B, Partial least squares discriminant analysis plot showing the covariance of all root sample groups. $\mathbf{C}$, Volcano plots presenting the number of spectral features that were statistically different with at least a fold change of 2 and a $P$ value $<0.05$. The first plot shows the lower abundance in CTRL roots and the higher abundance in roots that were colonized by SmR1. The second plot represents the lower abundance in CTRL roots and the higher abundance in SmR54 roots. The third plot represents the inoculated roots of SmR1 at lower abundance and the SmR54 at higher abundance. D, Box-and-whisker plots of four significant metabolites showing their relative abundances. 
of specific metabolites, it would be necessary to analyze specific plant or bacterial mutants, or both, blocked in the corresponding pathways. Previously, for example, we used this approach to assign changes in trehalose seen in soybean root hairs to the infecting bacterial symbiont (Brechenmacher et al. 2010).

\section{Allocation of metabolites in the leaves \\ from plants colonized by endophytic PGPB.}

The main focus of our work was to analyze and characterize the metabolites of PGPB-colonized roots. However, simultaneously, we took the opportunity to also examine the metabolome of the youngest, newly emerged sink leaf from the same set of plants used for the root analyses (the PLS-DA scores plot is shown in Figure 3). We, again, used six biological replicates for the leaf analyses. Perhaps surprisingly, the comparison of the spectra from the roots and leaves of similarly inoculated and uninoculated plants showed a degree of similarity and separation between plant tissues (Fig. 3). The first component in PLS-DA represented the separation between different types of tissues (root and leaf) from the plants. The second component showed no separation, since all these tissues are from the same plants.

The leaf metabolites that were significantly more abundant in either the CTRL, SmR1, or SmR54 plant tissues were identified (Supplementary Table S1). In contrast to roots, there were no

Table 1. Identified metabolites and pathways affected in Setaria viridis roots infected with either SmR1 or SmR54

\begin{tabular}{|c|c|c|c|c|}
\hline \multirow[b]{2}{*}{ Pathway/metabolite } & \multirow[b]{2}{*}{ KEGG ID } & \multicolumn{3}{|c|}{$\log _{2}(\mathrm{FC})$} \\
\hline & & SmR1 versus CTRL & SmR54 versus CTRL & SmR54 versus SmR1 \\
\hline \multicolumn{5}{|l|}{ Flavonoid biosynthesis } \\
\hline Methylpyranosyl glucoside ${ }^{\mathrm{b}}$ & & 5.10 & $6.67 * *$ & $2.33 *$ \\
\hline Hydroxybutyrate glucoside ${ }^{b}$ & & -0.05 & -2.24 & $-2.40 *$ \\
\hline Trihydroxyflavone $^{\mathrm{b}}$ & $\mathrm{C} 06563$ & -1.63 & -3.48 & $-2.86 *$ \\
\hline Dimethoxy-flavone & C11620 & 1.67 & -0.97 & $-3.34 * *$ \\
\hline Tetramethoxyflavanone $\mathrm{b}^{\mathrm{b}}$ & $\mathrm{C} 14472$ & 1.50 & & $-2.22 *$ \\
\hline Acetyl-prenylphenol glucoside ${ }^{\mathrm{b}}$ & $\mathrm{C} 04608$ & 1.79 & -1.88 & $-2.51 *$ \\
\hline Hydroxyflavanone glucoside ${ }^{b}$ & C16989 & $1.62 *$ & $1.76 *$ & -1.44 \\
\hline Dihydrochalcone glucoside & C01604 & -0.52 & & $-2.05 *$ \\
\hline Dihydroxy methoxyflavone glucoside ${ }^{b}$ & $\mathrm{C} 10381$ & 0.16 & & $-2.29 *$ \\
\hline Hydroxy dimethoxyflavone glucoside ${ }^{\mathrm{b}}$ & & 0.69 & & $-2.13 *$ \\
\hline Dihydroxyisoflavone malonyl glucoside ${ }^{b}$ & C16191 & $2.24 *$ & & -1.62 \\
\hline Trihydroxy trimethoxyflavone glucoside & & -0.69 & $-1.90 *$ & $-2.02 *$ \\
\hline $\begin{array}{l}\text { Trihydroxy-tetramethoxyflavone } \\
\text { glucoside }\end{array}$ & & -1.50 & $-\mathbf{3 . 1 8} *$ & \\
\hline \multicolumn{5}{|l|}{ Starch and sucrose metabolism } \\
\hline Glucose $\mathrm{e}^{\mathrm{b}}$ & $\mathrm{C} 00031$ & -0.48 & $-1.81 *$ & $-1.53 *$ \\
\hline Glucose phosphate ${ }^{b}$ & $\mathrm{C} 00103$ & $-1.30 *$ & $-3.02 * *$ & -1.65 \\
\hline Dissacharide ${ }^{\mathrm{b}}$ & C00089 & $-2.06 *$ & $-3.84 * *$ & -1.97 \\
\hline \multicolumn{5}{|l|}{ Cysteine and methionine metabolism } \\
\hline Sulfolactate ${ }^{\mathrm{b}}$ & C11537 & $-2.12 *$ & $-3.28 *$ & -0.75 \\
\hline Gutathione $^{\mathrm{b}}$ & $\mathrm{C} 00051$ & $-2.01 *$ & $-3.20 *$ & \\
\hline \multicolumn{5}{|l|}{ Pyrimidine metabolism } \\
\hline $\mathrm{UDP}^{\mathrm{b}}$ & $\mathrm{C} 00015$ & -1.50 & $-2.52 *$ & \\
\hline $\mathrm{UMP}^{\mathrm{b}}$ & $\mathrm{C} 00105$ & 0.38 & -2.56 & $-1.73 *$ \\
\hline \multicolumn{5}{|l|}{ Indole alkaloid biosynthesis } \\
\hline Norajmaline & C11810 & $8.65 *$ & $6.17 * *$ & $2.07 * *$ \\
\hline Ajmaline $^{\mathrm{b}}$ & $\mathrm{C} 06542$ & $8.65 *$ & $6.43 * *$ & $1.60 *$ \\
\hline \multicolumn{5}{|l|}{ Phenylpropanoid biosynthesis } \\
\hline Sinapoylglucose $\mathrm{b}^{\mathrm{b}}$ & $\mathrm{C} 01175$ & -1.15 & $-\mathbf{3 . 0 3} *$ & $-2.36 *$ \\
\hline Pimpinellin ${ }^{\mathrm{b}}$ & $\mathrm{C} 09285$ & $-2.08 *$ & $-3.49 *$ & \\
\hline \multicolumn{5}{|l|}{ Fatty acid biosynthesis } \\
\hline Heptose phosphate ${ }^{b}$ & $\mathrm{C} 07836$ & -0.24 & & $-1.51 *$ \\
\hline Hydroxyjasmonic acid glucoside ${ }^{b}$ & $\mathrm{C} 08558$ & $-2.08 *$ & $-3.18 *$ & -1.68 \\
\hline \multicolumn{5}{|l|}{ Pentose and glucuronate interconversions } \\
\hline Gulonate $\mathrm{b}^{\mathrm{c}}$ & C00800 & 0.11 & $-1.94 *$ & -2.22 \\
\hline \multicolumn{5}{|l|}{ Naphthalene degradation } \\
\hline Dihydroibenzothiophene & C14092 & 0.50 & $-1.59 *$ & -1.99 \\
\hline \multicolumn{5}{|l|}{ Puromycin biosynthesis } \\
\hline Puromycin aminonucleoside & $\mathrm{C} 01610$ & 3.09* & $3.89 *$ & $1.96 * *$ \\
\hline \multicolumn{5}{|l|}{ Salicylate degradation } \\
\hline Dihydroxybenzoate glucoside ${ }^{b}$ & C00628 & 0.13 & $-2.25 *$ & $-3.05 * *$ \\
\hline \multicolumn{5}{|l|}{ Aminobenzoate degradation } \\
\hline Dehydrodivanillate $^{\mathrm{b}}$ & $\mathrm{C} 18347$ & $-2.00 * *$ & $-2.60 *$ & -1.70 \\
\hline \multicolumn{5}{|l|}{ Pyruvate metabolism } \\
\hline Lactoylglutathione & $\mathrm{C} 03451$ & -1.13 & $-3.02 *$ & -1.68 \\
\hline \multicolumn{5}{|l|}{ Miscellaneous } \\
\hline Thiolutin/methylmalate & & -0.41 & -2.76 & $-2.56 *$ \\
\hline Sequoyitol $^{\mathrm{b}}$ & $\mathrm{C} 03365$ & $1.43 *$ & & $-2.15 * *$ \\
\hline Methylbutanoylapiosylhexose ${ }^{\mathrm{b}}$ & C11916 & $1.12 *$ & & $-1.84 *$ \\
\hline Bis(glycerophosphoglycerol) & $\mathrm{C} 03274$ & -0.65 & $-2.27 *$ & -1.96 \\
\hline
\end{tabular}

a The uninoculated (CTRL) plants were also analyzed for comparison. These metabolites had a significant fold change of at least 2 and $P$ value of $<0.05$ (shown in bold). The positive fold change is the upregulation number, while the negative fold change is the downregulation in abundance. KEGG $=$ Kyoto Encyclopedia of Genes and Genomes. One asterisk (*) means $P<0.05$ and two (**) $P<0.005$.

b Metabolites assigned by in-house reference standard tandem mass spectrometry performed under identical conditions. 
detectable differences in the abundance of flavonoid-like compounds in the leaf samples, consistent with the stronger expression of these compounds in roots (Webster et al. 1998). Similarly, consistent with the localization of photosynthesis and starch biosynthesis in leaves, compounds associated with these pathways were more abundant in leaves, irrespective of bacterial strain, with a $\log _{2}(\mathrm{FC})$ of $1.72,2.77$, and 1.56 for sucrose, ferulyl glucose, and hexose phosphate, respectively (Supplementary Table S1).

Of note, especially with regard to possible impact on plant growth promotion, we measured high levels of auxin, indole-3acetic acid (IAA), in the leaves of SmR1-inoculated plants, relative to those from the uninoculated control and SmR54inoculated plants (Supplementary Table S1). Earlier reports (Lugtenberg and Kamilova 2009; Monteiro et al. 2012; Spaepen and Vanderleyden 2011) implicated changes in phytohormone levels, especially auxin, as a possible mechanism to explain bacterial plant growth promotion. IAA has a variety of effects in plants, including impacting root branching and vascular development in the shoots (McSteen 2010). The main auxin biosynthesis pathway is activated when tryptophan converts to IAA in plants, and we found a greater abundance with a $\log _{2}(\mathrm{FC})$ of 2.08 in the SmR1-inoculated than the SmR54inoculated leaves (Supplementary Table S1). PGPB are capable of synthesizing auxin as well as other plant relevant hormones (Baca and Elmerich 2007; Dobbelaere et al. 2001; Fulchieri et al. 1993; Kramer and Bennett 2006; Spaepen and Vanderleyden 2011). Therefore, our LAESI-MS analysis correlates well with other studies regarding IAA from PGPB in the host. However, note that, based on measurements 3 weeks after inoculation, strain SmR54, lacking BNF ability, did promote plant growth, similar to strain SmR1 (Supplementary Fig. S3A). Hence, there is no correlation between the elevated presence of IAA, plant growth promotion, and the occurrence of BNF in the roots. It is equally likely that other metabolomic changes, more attributable to BNF, could impact IAA levels in the SmR1-inoculated plants without a measurable impact on plant growth. PGPB can produce auxin but, unlike leaves, significant levels of IAA or tryptophan were not found in the inoculated root segments analyzed, irrespective of BNF (Supplementary Table S1). Overall, even though the elevation of IAA in the leaves of SmR1-inoculated plants is interesting, we are unable to strongly argue it is playing a key role in bacterial plant growth promotion.

A variety of other metabolites were identified as significantly more abundant in either roots or leaves, relative to treatment (Supplementary Tables S2 and S3). These were analyzed by analyses of variance with $\mathrm{F}$ values ranging from 3 to 130 in SmR1 tissues (Supplementary Table S2) and 3 to 85 in SmR54 tissues (Supplementary Table S3). In addition, Supplementary Table S4 lists the identified metabolites that were significant between tissues of SmR1 and SmR54 with F values ranging from 3 to 212 .

\section{Metabolic pathways involved in plant growth promotion} associated with endophytic bacteria.

After identifying the significant metabolites (Tables 1, Supplementary Tables S1, S2, S3, and S4), their Kyoto Encyclopedia of Genes and Genomes (KEGG) identification numbers were used for pathway fold enrichment analyses in MetaboAnalyst 4.0 against the rice (Oryza sativa) library. Specifically, threefold enrichment graphs were created: i) SmR1 versus CTRL tissues (Supplementary Fig. S4), ii) SmR54 versus CTRL tissues (Supplementary Fig. S5), and iii) SmR1 versus SmR54 (Fig. 4). Comparing the SmR1 and CTRL samples, 36 metabolites were used for SmR1 analyses and 20 for the CTRL. Between SmR54 and CTRL samples, there were
28 compounds in the SmR54 and 34 in the CTRL. Additionally, 60 metabolites were used for SmR1 plant tissues and 28 compounds for SmR54. From these analyses, we detected pathways that had a range from one- to 35 -fold enrichment.

There was a total of 15 pathways enriched in the CTRL, 17 in the SmR1, and eight in the SmR54 roots. Metabolic pathways, including starch and sucrose metabolism, nitrogen metabolism, amino sugar metabolism, and chlorophyll metabolism were highly influenced in CTRL compared with SmR1 and SmR54 samples as expected (Supplementary Figs. S4 and S5). Even with only 2 weeks of growth, the CTRL and SmR54 plants were slightly $\mathrm{N}$ starved since a $\mathrm{N}$ source was not provided during growth. However, our previous measurements estimated that only approximately $7 \%$ of the daily nitrogen needs of the plant could be provided by inoculation with wild-type BNF bacteria (Pankievicz et al. 2015). However, this same study showed that plants grown with bacterial associations under mild nitrogenlimiting conditions behaved metabolically and physiologically like normal unstressed plants based on carbon-11 tracer experiments (Pankievicz et al. 2015). Therefore, one must be cautious in attributing specific metabolic differences only to BNF. These comparisons highlight just how difficult it is to have appropriate controls when comparing differing nutrient levels, since nutrient deprivation can have a variety of consequences.

The relative abundance of purine, zeatin, and riboflavin metabolites was significantly higher in the SmR1 and SmR54 inoculated roots relative to CTRL roots (Supplementary Figs. S4 and S5). There was approximately seven- to 11-fold enrichment of purine metabolism in the SmR1 and SmR54 roots relative to CTRL roots. An explanation for this is not obvious but may reflect a stimulation of localized plant metabolism, although we also cannot rule out elevation of these compounds due to plant growth. Perhaps more interesting is the elevation of both zeatin and riboflavin in bacteria-infected roots, irrespective of BNF (Supplementary Figs. S4 and S5). These data suggest a positive correlation between the elevation of these compounds and measurements of bacterial growth promotion. Zeatin (cytokinin) has a variety of effects on plant growth, including modulating root architecture (Aloni et al. 2006). Cytokinin is a key phytohormone involved in legume nodule

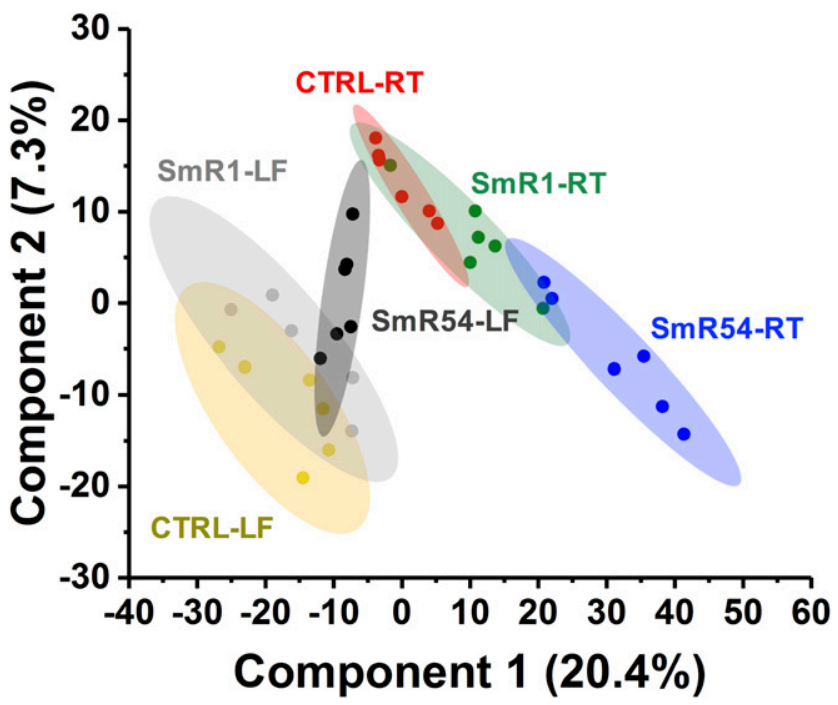

Fig. 3. Differences between spectra of uninoculated and inoculated plants, comparing the root and leaf samples. A partial least squares discriminant analysis scores plot shows the contrast between the spectra of leaf and root samples. Root segment spectra of uninoculated (CTRL) are in red, SmR1 are in green, and SmR54 are in blue. Leaf sample spectra of CTRL are in yellow, SmR1 are in light gray, and SmR54 are in dark gray. 
formation, and elevation in zeatin was detected during soybean root nodulation (Oldroyd and Downie 2008; Oldroyd et al. 2011; Stopka et al. 2017). There have also been reports in legumes in which rhizobia-produced riboflavin was shown to promote plant growth, but riboflavin can also have a variety of other effects (Kanu and Dakora 2012).

The pathway analyses between SmR1 versus SmR54 were of great interest in order to determine what pathways were affected by BNF (Fig. 4). A total of 11 pathways were detected in the SmR54 roots and there were 16 in the SmR1 plants. Similar LAESI-MS results using nodulated soybean plants (Agtuca et al. submitted) also found that zeatin, purine, and riboflavin metabolism were elevated in plants infected with fix ${ }^{+}$bacteria relative to those infected with a fix ${ }^{-}$strain; whereas, in plant roots infected with SmR54, pentose and glucuronate, indole alkaloid, pyrimidine, terpenoid biosynthesis, and sugar metabolism were enriched (Fig. 4).

\section{DISCUSSION}

The variable and sometimes high cost of $\mathrm{N}$ fertilizer (derived from fossil fuels) as well as the detrimental consequences of continued use of high levels of $\mathrm{N}$ fertilizer have led to efforts to enhance the ability to use BNF in nonlegume crops. Barriers to the widespread adoption of BNF in such cropping systems are those common to the agricultural use of all biological agents, such as variability of field-to-field efficacy, competition from endogenous soil organisms, ease of application, and others. An improved understanding of the molecular mechanisms by which PGPB stimulate plant growth, including the potential for $\mathrm{BNF}$ to mediate these effects, would contribute to solutions to these practical problems. Efforts to address the questions of molecular mechanism in our lab have included laboratory demonstration that high levels of BNF can be achieved in $S$. viridis when roots were inoculated with an $A$. brasilense strain specifically engineered to secrete ammonium (Pankievicz et al. 2015). In other work, we also demonstrated the advantages of adopting model grass species for studies of PGPB (do Amaral et al. 2016) and also conducted more detailed studies of bacterial genes essential for plant growth promotion (Alves et al. 2019). In the current study, we expanded these investigations by examining metabolic changes that occur at the localized sites of bacterial colonization of $S$. viridis roots, using LAESI-MS analysis.

Root colonization of $S$. viridis roots by fix ${ }^{+}$and fix endophytic bacteria results in a variety of complex and diverse metabolic changes.

As we demonstrated, bulk analysis of roots colonized by H. seropedicae SmR1 or SmR54, relative to uninoculated

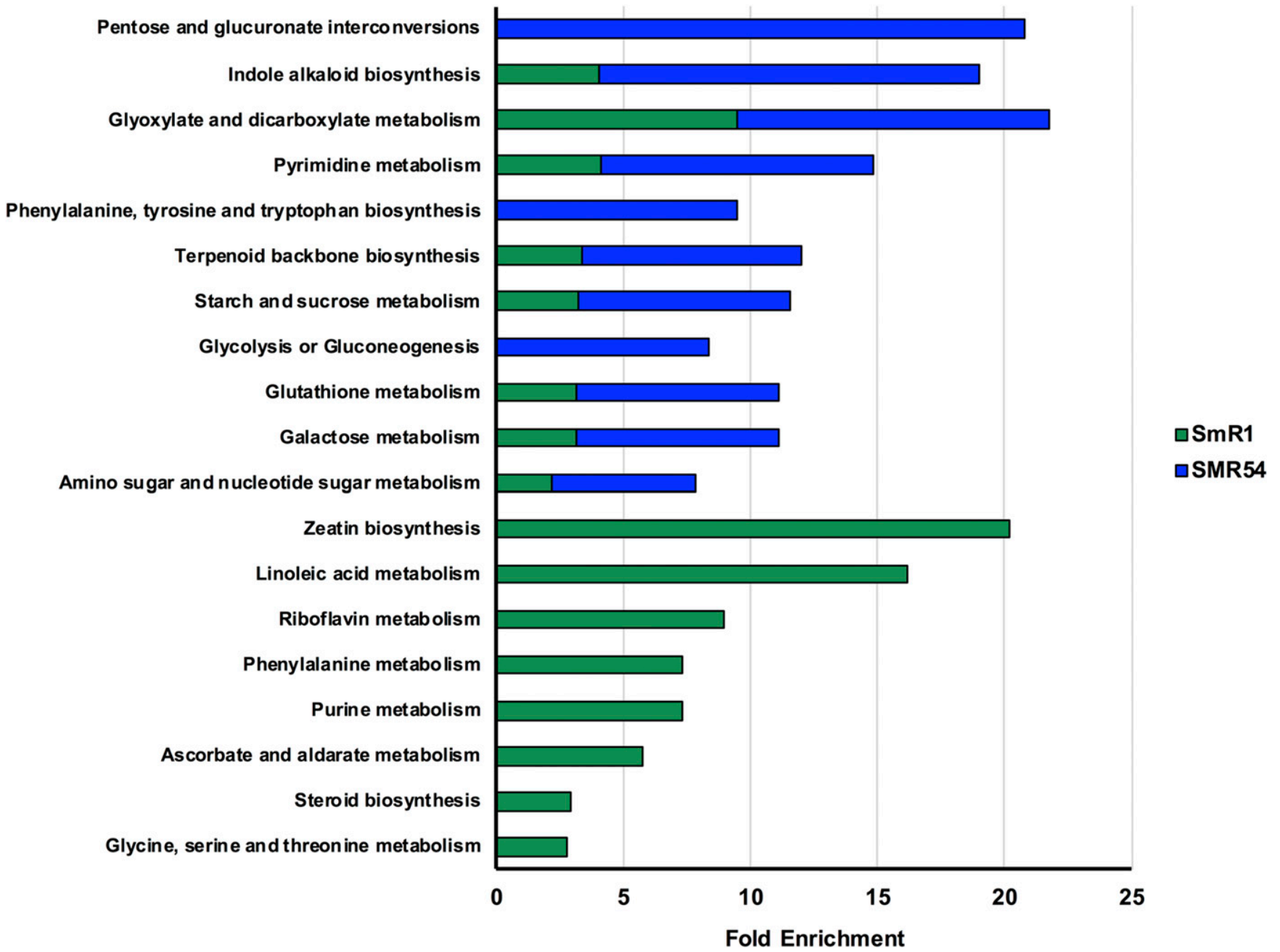

Fig. 4. Metabolic pathways significantly impacted by infection with SmR1 Herbaspirillum seropedicae (fix ${ }^{+}$, green) or with SmR54 H. seropedicae (fix ${ }^{-}$, blue). All the identified metabolites with a fold change $>2$ and a $P$ value $<0.05$ were used for the enrichment analysis, using the MetaboAnalyst 4.0 web resource, against the rice metabolite library as reference. These pathways highlight the importance of symbiosis, biological nitrogen fixation, growth promotion, and metabolism. The pathway analyses had a range of $P$ values from $8 \times 10^{-7}<P<6 \times 10^{-1}$. 
plants, failed to detect any significant differences. This is not surprising given that, judging by the distribution of bacterial infection in the root (as visualized by GFP expression), the great majority of the root is not in contact with the bacteria and, hence, may not be responding to any significant level. Dilution of the overall metabolite pool by these nonresponding tissues would likely overwhelm any localized responses due to bacterial infection. Thus, the use of an in-situ sampling method, such as LAESI-MS, was essential to our ability to detect changes in plant metabolism resulting from PGPB association. Indeed, by this method, there were a number of metabolites whose abundance changed significantly as a result of inoculation with either $H$. seropedicae $\mathrm{SmR} 1$ or SmR54 relative to the uninoculated plants. Collectively, the data clearly show that bacterial inoculation had a dramatic effect on metabolite abundance in general and that BNF also contributed significantly to changes in metabolism. Our findings are consistent with a variety of previous reports that used less-localized analyses to conclude that colonization by endophytic bacteria can dramatically affect the plant metabolome and transcriptome as well as growth (Aguiar et al. 2016; Dall'Asta et al. 2017; da Fonseca Breda et al. 2019; do Amaral et al. 2014; HaubergLotte et al. 2012; Matilla et al. 2007; Pankievicz et al. 2015, 2016; Shidore et al. 2012; Vacheron et al. 2013). Specifically, Brusamarello-Santos et al. (2017) and Sarkar and ReinholdHurek (2014) obtained somewhat similar results when there were induced changes in abundance and expression between plants that were inoculated with a fix ${ }^{+}$bacterium relative to a fix $^{-}$mutant strain deleted in nifA, a transcriptional activator for nif genes.

Our previous study used ${ }^{13} \mathrm{~N}_{2}$ labeling to show that dual inoculation of Setaria viridis plants with wild-type $H$. seropedicae and A. brasilense could provide roughly $7 \%$ of the $\mathrm{N}$ needs of the plant (Pankievicz et al. 2015). However, inoculation with a hyper-fixing, ammonium-excreting mutant of A. brasilense provided up to $100 \%$ of the plant's N needs. This latter result demonstrated the potential for BNF by PGPB to support plant growth. In this same study, changes in general metabolite classes (e.g., amino acids) were determined using carbon-11 radiotracers. The results documented significant shifts in metabolic pools due both to bacterial inoculation and BNF (Pankievicz et al. 2015). An analysis in maize plants inoculated with $H$. seropedicae wild-type $\operatorname{SmR} 1\left(\right.$ fix $^{+}$) and the BNF-defective mutant strain SmR54 (fix ${ }^{-}$) also documented shifts in both starch and sucrose metabolism in the fix ${ }^{+}$plants relative to those not fixing nitrogen (Brusamarello-Santos et al. 2017). Although both these studies provide results consistent with our findings, the use of LAESI-MS on the specific sites of bacterial colonization provides more specific data and a larger set of differentially affected metabolites.

Although inoculation clearly impacted plant metabolism, a few compounds appeared more abundant in the uninoculated roots. One example is hydroxyjasmonic acid glucoside, which is a component of jasmonic acid biosynthesis (Koch et al. 1997). Jasmonic acid is a well-characterized phytohormone playing key roles in plant development as well as in the defense response to wounding and abiotic and biotic stress (Koo 2018; Liechti and Farmer 2002; Wasternack 2007; Wasternack and Hause 2013). The elevation of JA-related compounds in the CTRL roots may be due to nitrogen deprivation, but then we would also expect this in the SmR54-infected roots, which was not seen in our analysis. Hydroxyjasmonic acid glucoside is a glycosidic conjugate formed during jasmonic acid biosynthesis (Widemann et al. 2013) and its formation switches off jasmonic acid signaling (Miersch et al. 2008). However, we cannot rule out other functions for this compound in plant metabolism. It is interesting to speculate that the higher abundance of hydroxyjasmonic acid glucoside in CTRL roots is a reflection of the lack of infection, with hydrolysis of this compound occurring in roots upon PGPB infection. The interplay of specific phytohormones, plant defense pathways, and plant growth is complex, but could be a significant contributor to PGPBinduced plant growth.

Sequoyitol was detected and was more abundant in the SmR1 roots, with a $\log _{2}(\mathrm{FC})$ of 2.15 and 1.43 , relative to the SmR54 and CTRL root samples (Table 1). However, there is little data on the function of this metabolite in plants. Sequoyitol is a cyclitol, which may function as an osmolyte or osmoprotectant (Ford 1984) as well as a carbon storage compound in plants (Richter and Popp 1992). It was shown that cyclitols increase in abundance in both legumes and nonlegumes in response to drought and other abiotic stresses (Ford 1984; Guo and Oosterhuis 1995, 1997; Keller and Ludlow 1993; Streeter et al. 2001; Wanek and Richter 1997). Regarding nitrogen-fixing, symbiotic associations, cyclitols, including sequoyitol, are abundant in the infected cells within the central region of soybean root nodules (Phillips et al. 1982; Streeter 1980; Streeter and Bosler 1976). However, their specific role in nodule metabolism remains undefined.

\section{Intersections between the metabolome of PGPB-infected roots and plant innate immunity.}

Roots colonized by the SmR54 strain had an abundance of ajmaline and norajmaline, relative to roots infected by the wildtype SmR1 or CTRL samples. Ajmaline, norajmaline, and related compounds (vinblastine, vincristine, vindoline) are involved in terpenoid indole alkaloid (TIA) biosynthesis that occurs in a jasmonate-responsive manner and has been studied in a variety of plant systems, including Rauvolfia serpentine and Catharanthus roseus (Facchini and De Luca 2008; Facchini and St-Pierre 2005; Guirimand et al. 2011; St-Pierre et al. 1999). These alkaloid metabolites are low-molecular weight, heterocyclic compounds and have been studied in large part due to their pharmacological activities (van Der Heijden et al. 2004; Verma et al. 2012). However, these compounds are present in plants in low amounts making them expensive to purify from plant tissues. Studies have focused on finding ways to increase the accumulation of these metabolites for therapeutic and pharmaceutical uses (van Der Heijden et al. 2004). However, they appear to be absent in most plants, including the wellstudied model system Arabidopsis (Van Moerkercke et al. 2013). There are examples, nonetheless, in which infection by fungal endophytes, such as those infecting C. roseus, significantly enhances terpenoid biosynthesis (Kumar et al. 2013; Pandey et al. 2016; Tiwari et al. 2013). In these specific situations, the presence of the fungal endophyte also significantly affects plant growth as well as stress tolerance. Hence, it is intriguing that higher levels of such terpenoid compounds were found in Setaria roots after PGPB inoculation, although clearly the data do not establish cause and effect between these compounds and growth promotion. However, here again, a perfect correlation is not found, since it was the roots infected by the SmR54 strain that showed the highest elevation of TIA abundance (i.e., seven- to tenfold), while both the SmR1- and SmR54-inoculated plants showed measurable effects on plant growth.

If one reads reviews on legume-rhizobial symbiosis as well as articles describing associative (PGPB) associations, one might get the impression that the former is quite complex while the latter can be explained by relatively simple changes (e.g., in auxin or cytokinin levels) (Chagas et al. 2018; Lugtenberg and Kamilova 2009; Oldroyd and Downie 2008; Oldroyd et al. 2011). However, a major conclusion from our study is that colonization of Setaria roots by $H$. seropedicae elicits a large 
variety of complex metabolic changes, suggesting the bacteria may have the ability to exploit the plant in specific ways to support its growth and stability. In this way, the interaction of PGPB with plants would not be that different, in a general sense, from other plant-microbe interactions, many of which have been studied in far greater detail than PGPB-plant associations. With regard to plant symbionts, PGPB are also similar to rhizobia in that they can colonize the root to quite high numerical levels without the induction of a visible plant defense response. Our metabolomics data confirms the absence of many metabolites one would associate with plant defense, although it is not clear that PGPB are totally benign to the plant. Since our laboratory has also conducted metabolite profiling of soybean root nodules, we compared the results of these studies (Agtuca et al. submitted). There are clear similarities. For example, both systems show a significant impact on auxin, purine, zeatin, riboflavin, and starch and sucrose metabolism as well as induction of flavonoid accumulation. We are still in the early stages of understanding the complexity of PGPB infection, establishment, and function in plants, especially in comparison with the wealth of knowledge available on the legume symbiosis.

\section{MATERIALS AND METHODS}

\section{Bacterial culture conditions.}

H. seropedicae SmR1 (fix ${ }^{+}$) and SmR54 (fix $\left.{ }^{-}\right)$strains were grown in NFbHP-malate liquid medium that contained $20 \mathrm{ml}$ of $\mathrm{NH}_{4} \mathrm{Cl}$ per liter as the nitrogen source (Klassen et al. 1997) at $30^{\circ} \mathrm{C}$ and $130 \mathrm{rpm}$, overnight. Streptomycin at a final concentration of $80 \mu \mathrm{g} \mathrm{ml}^{-1}$ and kanamycin at a final concentration of $200 \mu \mathrm{g} \mathrm{ml}^{-1}$ were added to the medium. Once the cultures reached an optical density at $600 \mathrm{~nm}\left(\mathrm{OD}_{600}\right)$ of $1.0\left(10^{8}\right.$ cells $\mathrm{ml}^{-1}$ ), the bacteria were pelleted and were washed three times with $0.9 \%$ of $\mathrm{NaCl}$ solution. The bacterial suspension was diluted to $10^{7}$ cells $\mathrm{ml}^{-1}$ and $1 \mathrm{ml}$ per seedling was used for inoculation.

\section{Plant growth and inoculation.}

Setaria viridis A10.1 seeds were pretreated with sulfuric acid for $15 \mathrm{~min}$. Seeds were then rinsed with water and were sterilized with $1 \%$ (vol/vol) bleach plus $0.1 \%$ (vol/vol) Tween 20 for $3 \mathrm{~min}$ and were washed $3 \times$ with sterile deionized water. The seeds were transferred and were germinated on Hoagland's solution on $1 \%(\mathrm{wt} / \mathrm{vol})$ phytagel agar (Hoagland and Arnon 1950). Seeds were incubated at $30^{\circ} \mathrm{C}$ for 1 day in the dark, followed by 2 days in the light. At 3 days postgermination, seedlings were soaked in either SmR1 or SmR54 bacterial suspensions for $30 \mathrm{~min}$. The control seedlings were soaked in sterile Hoagland's solution with no inoculum. Upon inoculation, all seedlings from different sample groups (CTRL, SmR1, SmR54) were planted in soil comprised of a 3:1 Turface/vermiculite mixture, respectively. The Setaria plants grew for 2 weeks postinoculation, after which they were either observed by microscopy or were rapidly frozen in liquid nitrogen for subsequent extraction for bulk or LAESI-MS metabolomic analyses. A subset of the roots was also used to measure the level of bacterial colonization. Some plants from the same batch were grown for an additional week, in order to quantify plant growth promotion.

\section{Confocal and fluorescence imaging of root colonization.}

H. seropedicae $\mathrm{SmR} 1\left(\mathrm{fix}^{+}\right)$and $\mathrm{SmR} 54$ (fix ${ }^{-}$), both constitutively expressing GFP, were used to identify areas of bacterial colonization in the roots of $S$. viridis A10.1. The control and inoculated roots were placed on a slide separately with a drop of water, which was covered with a glass coverslip to view, either using a laser scanning confocal microscope or fluorescence microscope (Zeiss Axiovert 200M) with Leica DFC290 color camera. The entire roots were closely examined, specifically at the upper (close to the seed) and lower parts (elongation zone), in order to define a segment with the highest level of endophytic bacterial colonization (as judged by GFP). Bright and fluorescence images were obtained as Z-stack and were overlaid in the Metamorph v.7.8.12 software program. After imaging and examining each entire root by fluorescence microscope, the roots that had the highest level of endophytic bacterial colonization were selected and were cut into segments. There was a total of 20 root segments of approximately $1 \mathrm{~cm}$ in length that were harvested from 10 different roots, were frozen in liquid nitrogen, and were stored at $-80^{\circ} \mathrm{C}$ until LAESI analysis. Each segment represents a biological replicate. A similar number of root segments from roughly the same regions of the root were harvested from uninoculated plants to serve as the control. Shoots from 10 different plants, but from the same set of plants used to harvest the root tissue, were also harvested, frozen in liquid nitrogen, and stored at $-80^{\circ} \mathrm{C}$ until LAESI analysis.

\section{LAESI-MS instrumentation.}

The instrumentation was similar as for our previous paper (Stopka et al. 2017). A mid-IR laser (IR OpoletteHE 2731, Opotek, Carlsbad, CA, U.S.A.) operating at $2.94 \mu \mathrm{m}$ wavelength and $20 \mathrm{~Hz}$ repetition rate was used for direct ablation of the root segments. The laser beam was steered using goldcoated mirrors through a 50-mm focal length plano-convex $\mathrm{CaF}_{2}$ focusing lens. The frozen root segments were placed onto a microscope slide on an automated XY stage (MLS203-1, Thorlabs, Newton, NJ, U.S.A.) in direct line with the focused laser beam. An ablation plume of neutrals was produced as the root segment was targeted, which was then ionized by an electrospray, and the ions where sampled by the mass spectrometer, which was operating in negative ion mode (Fig. 1). The electrospray solvent composition was $2: 1(\mathrm{vol} / \mathrm{vol}) \mathrm{MeOH}$ : $\mathrm{CHCl}_{3}$ and was dispensed at a flow rate of $500 \mathrm{nl} / \mathrm{min}$ through a stainless steel emitter held at $2.7 \mathrm{kV}$ spray voltage. For targeted ablation, a side microscope (AM4815ZTL, Dino-lite, Torrance, CA, U.S.A.) was used to ensure the whole root segment was ablated and the sample was correctly positioned using the automated stage.

\section{Setaria viridis bulk analyses.}

Approximately $10 \mathrm{mg}$ of frozen root tissue from the control and inoculated plants were homogenized for LAESI-MS bulk analyses. The tissues were placed into 2-ml Eppendorf tubes and were suspended with $40 \mu \mathrm{l}$ of deionized water. The samples were probe-sonicated (QSonica Q125, Newton, CT, U.S.A.) for $30 \mathrm{~s}$ with 1-s pulse durations, at an amplitude of $30 \%$, while on ice. Approximately $10 \mu \mathrm{l}$ of the sonicated material was placed on a glass microscope slide for LAESI-MS analysis.

Metabolic profiling of plant tissues and bacterial pellets.

After fluorescence imaging, the harvested, frozen, root segments with the highest endophytic colonization (SmR1 and SmR54) and uninoculated segments $(n=6)$ were analyzed by LAESI-MS. These root segments were used without cryosectioning before the analyses. For the leaf analyses from the inoculated and uninoculated plants, the frozen leaves (same set of plants as analyzed from the root segments by fluorescence imaging) were observed, and out of the three developed leaves on each plant, the youngest, newly emerged sink leaf was selected, was cut to approximately $1 \mathrm{~cm}$ in length, and was used for in-situ metabolic profiling. The frozen selected tissues, including the sink leaf and root segments, were placed on a microscope slide and, $2 \mathrm{~s}$ later, $2 \mu \mathrm{l}$ of sterile water was added on top of the root segments in order to have higher water content for LAESI-MS ablation. Ten to $20 \mathrm{~s}$ after the samples were 
placed on the microscope slide, the leaves and the hydrated root segments were analyzed by LAESI-MS. A laser energy of approximately $1.5 \mathrm{~mJ}$ per pulse with a $20 \mathrm{~Hz}$ repetition rate was used to ablate the plant tissues in a raster formation.

Free-living cultures of $H$. seropedicae $\mathrm{SmR} 1$ (fix $^{+}$) wild-type and SmR54 fix ${ }^{-}$mutant strains were grown in the appropriate medium, as stated in the bacterial culture conditions section. These bacteria were then subcultured and were grown overnight in NFbHP-malate medium that had $20 \mathrm{ml}$ of $\mathrm{NH}_{4} \mathrm{Cl}$ per liter as a nitrogen source without antibiotics until reaching an $\mathrm{OD}_{600}=$ $1.0\left(10^{8}\right.$ cells $\left.\mathrm{ml}^{-1}\right)$. The bacterial cultures were centrifuged at $800 \times g$ for 2 min and were washed with sterile water. This was repeated a total of three times. After washing, the bacterial pellets were resuspended in $20 \mu \mathrm{l}$ of deionized water. The suspended pellet $(10 \mu \mathrm{l})$ was placed onto a microscope glass slide. Six biological replicates of the suspended pellets were analyzed by LAESI-MS.

\section{Biomass measurements and bacterial colonization assay.}

Plant roots at 2 weeks after inoculation were used to measure the level of bacterial colonization. Fresh roots were macerated in $1 \mathrm{ml}$ of $0.9 \%$ (wt/vol) $\mathrm{NaCl}$ solution. The homogenized roots were then serially diluted and $10 \mu \mathrm{l}$ of the final dilution were plated on solid NFbHP-malate medium with addition of the respective antibiotics. Plates were incubated for 3 days at $30^{\circ} \mathrm{C}$ and $\mathrm{CFU}$ were counted and converted into CFU per gram of fresh tissue.

The remaining plants grown for 3 weeks after inoculation were dried completely in a $45^{\circ} \mathrm{C}$ incubator for biomass measurements. Roots and shoots were weighed separately. Total biomass was calculated by summing the two dry-weight measurements.

\section{Metabolite identification.}

In order to obtain a library of $S$. viridis root and leaf metabolites, 10 plants were grown as described above, and their leaves and whole roots were harvested, were flash frozen in liquid nitrogen, and were stored at $-80^{\circ} \mathrm{C}$ until use. Approximately $1 \mathrm{~g}$ of the frozen root and leaf tissues were ground separately by mortar and pestle in liquid nitrogen until a fine powder was obtained. The ground samples were transferred to 1.5-ml Eppendorf tubes and were resuspended in 2:1 ( $\mathrm{vol} / \mathrm{vol})$ chloroform/methanol solution. The samples were vortexed and were centrifuged at $5,000 \times g$ for $5 \mathrm{~min}$ at room temperature. Then, the supernatant was transferred to a new 1.5-ml Eppendorf tube. The samples were centrifuged two more times in order to have a clear supernatant extract. These extracts were then transferred to a 500- $\mu$ l Hamilton syringe for ESI-MS/MS analyses. A syringe pump was used to drive the syringe that included the prepared lysates. In addition, the syringe was connected to a stainless-steel emitter (MT320-50-5-5; New Objective, Woburn, MA, U.S.A.) through a fused silica tube. The flow rate of the syringe pump was at $500 \mathrm{nl} / \mathrm{min}$ and the prepared solution was sprayed by employing a spraying voltage of $-2,200 \mathrm{~V}$ to the emitter. A quadrupole-time-of-flight mass spectrometer (Synapt G2S; Waters, Milford, MA, U.S.A.) was used to analyze the generated ions. Significant metabolites previously detected by LAESI-MS were chosen for MS/MS by collision induced dissociation with collision energies from 10 to $50 \mathrm{eV}$. The metabolite standard initiative levels for metabolite identification were implemented for peak assignments. All steps for metabolite identification were adapted from our previous study (Stopka et al. 2017).

\section{Data and statistical analyses.}

After LAESI-MS analyses, the raw mass spectra from plant samples and bacterial pellets with six biological replicates were processed by averaging ten MS scans and subtracting the background from equal numbers of ESI-only scans. The processed data were analyzed by MetaboAnalyst 4.0, a web-based metabolomic processing software, using univariate, multivariate, and hierarchical clustering statistical approaches. The data were normalized as described in the supporting methods from Stopka et al. (2017). Heat maps, PLS-DA scores and loading plots, and box-and-whisker plots were constructed by MetaboAnalyst 4.0. Volcano plots were also generated for all detected ions and the significant ions with a $P<0.05$ and a $\mathrm{FC}>2$ were highlighted, based on Student's $t$ test. The pathway analyses were conducted by MetaboAnalyst 4.0. The rice (Oryza sativa) pathways were downloaded from the KEGG database and were used in the pathway enrichment analysis as the reference set.

\section{ACKNOWLEDGMENTS}

We thank F. Baker and A. Jurkevich from the Molecular Cytology Core at the C. S. Bond Life Sciences Center, the University of Missouri and W. Chrisler from The Environmental Molecular Sciences Laboratory for their help on the microscope images. We acknowledge M. Cotta and T. Pellizzaro Pereira for help with growing Setaria plants. We thank J. Robil for the graphic design of Figure 1.

\section{LITERATURE CITED}

Aguiar, N., Medici, L., Olivares, F., Dobbss, L., Torres-Netto, A., Silva, S., Novotny, E., and Canellas, L. 2016. Metabolic profile and antioxidant responses during drought stress recovery in sugarcane treated with humic acids and endophytic diazotrophic bacteria. Ann. Appl. Biol. 168: 203-213.

Aloni, R., Aloni, E., Langhans, M., and Ullrich, C. I. 2006. Role of cytokinin and auxin in shaping root architecture: Regulating vascular differentiation, lateral root initiation, root apical dominance and root gravitropism. Ann. Bot. 97:883-893.

Alves, L. P. S., do Amaral, F. P., Kim, D., Bom, M. T., Gavídia, M. P., Teixeira, C. S., Holthman, F., de Oliveira Pedrosa, F., de Souza, E. M. and Chubatsu, L. S. 2019. Importance of poly-3-hydroxybutyrate (PHB) metabolism to the ability of Herbaspirillum seropedicae to promote plant growth. Appl. Environ. Microbiol. DOI: 10.1128/AEM.02586-18. Published online.

Baca, B., and Elmerich, C. 2007. Microbial production of plant hormones. Pages 113-143 in: Associative and endophytic nitrogen-fixing bacteria and cyanobacterial associations. Springer, Dordrecht, The Netherlands.

Balachandar, D., Sandhiya, G., Sugitha, T., and Kumar, K. 2006. Flavonoids and growth hormones influence endophytic colonization and in planta nitrogen fixation by a diazotrophic Serratia sp. in rice. World J. Microbiol. Biotechnol. 22:707-712.

Brechenmacher, L., Lei, Z., Libault, M., Findley, S., Sugawara, M. Sadowsky, M. J., Sumner, L. W., and Stacey, G. 2010. Soybean metabolites regulated in root hairs in response to the symbiotic bacterium Bradyrhizobium japonicum. Plant Physiol. 153:1808-1822.

Brusamarello-Santos, L. C., Gilard, F., Brulé, L., Quilleré, I., Gourion, B., Ratet, P., Maltempi de Souza, E., Lea, P. J., and Hirel, B. 2017. Metabolic profiling of two maize (Zea mays L.) inbred lines inoculated with the nitrogen fixing plant-interacting bacteria Herbaspirillum seropedicae and Azospirillum brasilense. PLoS One 12:e0174576.

Chagas, F. O., Pessotti, R. C., Caraballo-Rodríguez, A. M., and Pupo, M. T. 2018. Chemical signaling involved in plant-microbe interactions. Chem Soc. Rev. 47:1652-1704.

da Fonseca Breda, F. A., da Silva, T. F. R., dos Santos, S. G., Alves, G. C., and Reis, V. M. 2019. Modulation of nitrogen metabolism of maize plants inoculated with Azospirillum brasilense and Herbaspirillum seropedicae. Arch. Microbiol. 201:547-558.

Dall'Asta, P., Pereira, T. P., Do Amaral, F. P., and Arisi, A. C. M. 2017. Tools to evaluate Herbaspirillum seropedicae abundance and nifH and rpoC expression in inoculated maize seedlings grown in vitro and in soil. Plant Growth Regul. 83:397-408.

do Amaral, F. P., Agtuca, B. J., and Stacey, G. 2017. Setaria root-microbe interactions. Pages 239-250 in: Genetics and Genomics of Setaria, Springer, Cham, Switzerland.

do Amaral, F. P., Bueno, J. C. F., Hermes, V. S., and Arisi, A. C. M. 2014 Gene expression analysis of maize seedlings (DKB240 variety) inoculated with plant growth promoting bacterium Herbaspirillum seropedicae. Symbiosis 62:41-50. 
do Amaral, F. P., Pankievicz, V. C., Arisi, A. C. M., de Souza, E. M., Pedrosa, F., and Stacey, G. 2016. Differential growth responses of Brachypodium distachyon genotypes to inoculation with plant growth promoting rhizobacteria. Plant Mol. Biol. 90:689-697.

Dobbelaere, S., Croonenborghs, A., Thys, A., Ptacek, D., Vanderleyden, J., Dutto, P., Labandera-Gonzalez, C., Caballero-Mellado, J., Aguirre, J. F., and Kapulnik, Y. 2001. Responses of agronomically important crops to inoculation with Azospirillum. Funct. Plant Biol. 28:871-879.

Dobermann, A. 2007. Nutrient use efficiency: Measurement and management. Pages 1-28 in: Fertilizer Best Management Practices. A. Krauss, K. Isherwood, and P. Heffer, eds. International Fertilizer Industry Association, Paris.

Edmonds, D. E., Tubaña, B. S., Kelly, J. P., Crain, J. L., Edmonds, M. D., Solie, J. B., Taylor, R. K., and Raun, W. R. 2013. Maize grain yield response to variable row nitrogen fertilization. J. Plant Nutr. 36:1013-1024

Facchini, P. J., and De Luca, V. 2008. Opium poppy and Madagascar periwinkle: Model non-model systems to investigate alkaloid biosynthesis in plants. Plant J. 54:763-784.

Facchini, P. J., and St-Pierre, B. 2005. Synthesis and trafficking of alkaloid biosynthetic enzymes. Curr. Opin. Plant Biol. 8:657-666.

Falcone Ferreyra, M. L., Rius, S. P., and Casati, P. 2012. Flavonoids: Biosynthesis, biological functions, and biotechnological applications. Front. Plant Sci. 3:222.

Faoro, H., Rene Menegazzo, R., Battistoni, F., Gyaneshwar, P., do Amaral, F. P., Taulé, C., Rausch, S., Gonçalves Galvão, P., de Los Santos, C. Mitra, S., Heijo, G., Sheu, S. Y., Chen, W. M., Mareque, C., Zibetti Tadra-Sfeir, M., Ivo Baldani, J., Maluk, M., Paula Guimarães, A., Stacey, G., de Souza, E. M., Pedrosa, F. O., Magalhães Cruz, L., and James, E. K. 2017. The oil-contaminated soil diazotroph Azoarcus olearius DQS $-4^{\mathrm{T}}$ is genetically and phenotypically similar to the model grass endophyte Azoarcus sp. BH72. Environ. Microbiol. Rep. 9:223-238.

Fisher, L. H., Han, J., Corke, F. M., Akinyemi, A., Didion, T., Nielsen, K. K., Doonan, J. H., Mur, L. A., and Bosch, M. 2016. Linking dynamic phenotyping with metabolite analysis to study natural variation in drought responses of Brachypodium distachyon. Front. Plant Sci. 7:1751.

Ford, C. W. 1984. Accumulation of low molecular weight solutes in waterstressed tropical legumes. Phytochemistry 23:1007-1015.

Franche, C., Lindström, K., and Elmerich, C. 2009. Nitrogen-fixing bacteria associated with leguminous and non-leguminous plants. Plant Soil 321:35-59.

Fulchieri, M., Lucangeli, C., and Bottini, R. 1993. Inoculation with Azospirillum lipoferum affects growth and gibberellin status of corn seedling roots. Plant Cell Physiol. 34:1305-1309.

Galloway, J. N., Dentener, F. J., Capone, D. G., Boyer, E. W., Howarth, R. W., Seitzinger, S. P., Asner, G. P., Cleveland, C. C., Green, P., Holland, E. A., Karl, D. M., Michaels, A. F., Porter, J. H., Townsend, A. R., and Vöosmarty, C. J. 2004. Nitrogen cycles: Past, present, and future. Biogeochemistry 70:153-226.

Galloway, J. N., Townsend, A. R., Erisman, J. W., Bekunda, M., Cai, Z., Freney, J. R., Martinelli, L. A., Seitzinger, S. P., and Sutton, M. A. 2008. Transformation of the nitrogen cycle: Recent trends, questions, and potential solutions. Science 320:889-892.

Gemperline, E., Keller, C., and Li, L. 2016. Mass spectrometry in plantomics. Anal. Chem. 88:3422-3434.

Godfray, H. C. J., Beddington, J. R., Crute, I. R., Haddad, L., Lawrence, D. Muir, J. F., Pretty, J., Robinson, S., Thomas, S. M., and Toulmin, C. 2010. Food security: the challenge of feeding 9 billion people. Science 327:812-818.

Gough, C., Galera, C., Vasse, J., Webster, G., Cocking, E. C., and Dénarié, J. 1997. Specific flavonoids promote intercellular root colonization of Arabidopsis thaliana by Azorhizobium caulinodans ORS571. Mol. Plant-Microbe Interact 10:560-570.

Guirimand, G., Guihur, A., Poutrain, P., Héricourt, F., Mahroug, S., StPierre, B., Burlat, V., and Courdavault, V. 2011. Spatial organization of the vindoline biosynthetic pathway in Catharanthus roseus. J. Plant Physiol. 168:549-557.

Guo, C., and Oosterhuis, D. M. 1995. Pinitol occurrence in soybean plants as affected by temperature and plant growth regulators. J. Exp. Bot. 46: 249-253.

Guo, C., and Oosterhuis, D. M. 1997. Effect of water-deficit stress and genotypes on pinitol occurrence in soybean plants. Environ. Exp. Bot. 37:147-152.

Hardoim, P. R., van Overbeek, L. S., and Elsas, J. D. 2008. Properties of bacterial endophytes and their proposed role in plant growth. Trends Microbiol. 16:463-471

Hauberg-Lotte, L., Klingenberg, H., Scharf, C., Böhm, M., Plessl, J., Friedrich, F., Völker, U., Becker, A., and Reinhold-Hurek, B. 2012. Environmental factors affecting the expression of pilAB as well as the proteome and transcriptome of the grass endophyte Azoarcus sp. strain BH72. PLoS One 7:e30421.
Hoagland, D. R., and Arnon, D. I. 1950. The water-culture method for growing plants without soil. College of Agriculture, University of California, Berkeley, CA, U.S.A.

Kanu, S. A., and Dakora, F. D. 2012. Effect of $\mathrm{N}$ and $\mathrm{P}$ nutrition on extracellular secretion of lumichrome, riboflavin and indole acetic acid by N 2-fixing bacteria and endophytes isolated from Psoralea nodules. Symbiosis 57:15-22.

Keller, F., and Ludlow, M. 1993. Carbohydrate metabolism in droughtstressed leaves of pigeonpea (Cajanus cajan). J. Exp. Bot. 44:1351-1359.

Klassen, G., Pedrosa, F., Souza, E., Funayama, S., and Rigo, L. 1997. Effect of nitrogen compounds on nitrogenase activity in Herbaspirillum seropedicae SMR1. Can. J. Microbiol. 43:887-891.

Koch, T., Bandemer, K., and Boland, W. 1997. Biosynthesis of cisjasmone: A pathway for the inactivation and the disposal of the plan stress hormone jasmonic acid to the gas phase? Helv. Chim. Acta 80: 838-850.

Koo, A. J. 2018. Metabolism of the plant hormone jasmonate: A sentinel for tissue damage and master regulator of stress response. Phytochem. Rev 17:51-80.

Kramer, E. M., and Bennett, M. J. 2006. Auxin transport: A field in flux. Trends Plant Sci. 11:382-386.

Krause, A., Ramakumar, A., Bartels, D., Battistoni, F., Bekel, T., Boch, J., Böhm, M., Friedrich, F., Hurek, T., Krause, L., Linke, B., McHardy, A. C., Sarkar, A., Schneiker, S., Syed, A. A., Thauer, R., Vorhölter, F. J., Weidner, S., Pühler, A., Reinhold-Hurek, B., Kaiser, O., and Goesmann, A. 2006. Complete genome of the mutualistic, $\mathrm{N}_{2}$-fixing grass endophyte Azoarcus sp. strain BH72. Nat. Biotechnol. 24:1384-1390.

Kumar, A., Patil, D., Rajamohanan, P. R., and Ahmad, A. 2013. Isolation, purification and characterization of vinblastine and vincristine from endophytic fungus Fusarium oxysporum isolated from Catharanthus roseus. PLoS One 8:e71805.

Kumar, R., Bohra, A., Pandey, A. K., Pandey, M. K., and Kumar, A. 2017. Metabolomics for plant improvement: Status and prospects. Front. Plant Sci. 8:1302.

Liechti, R., and Farmer, E. E. 2002. The jasmonate pathway. Science 296 1649-1650.

Liu, C.-W., and Murray, J. D. 2016. The role of flavonoids in nodulation host-range specificity: An update. Plants Basel 5:33.

Lodwig, E. M., Leonard, M., Marroqui, S., Wheeler, T. R., Findlay, K., Downie, J. A., and Poole, P. S. 2005. Role of polyhydroxybutyrate and glycogen as carbon storage compounds in pea and bean bacteroids. Mol. Plant-Microbe Interact 18:67-74

Lugtenberg, B., and Kamilova, F. 2009. Plant-growth-promoting rhizobacteria. Annu. Rev. Microbiol. 63:541-556.

Marin, A. M., Souza, E. M., Pedrosa, F. O., Souza, L. M., Sassaki, G. L., Baura, V. A., Yates, M. G., Wassem, R., and Monteiro, R. A. 2013. Naringenin degradation by the endophytic diazotroph Herbaspirillum seropedicae SmR1. Microbiology 159:167-175.

Matilla, M. A., Espinosa-Urgel, M., Rodríguez-Herva, J. J., Ramos, J. L., and Ramos-González, M. I. 2007. Genomic analysis reveals the major driving forces of bacterial life in the rhizosphere. Genome Biol. 8:R179.

McSteen, P. 2010. Auxin and monocot development. Cold Spring Harb. Perspect. Biol. 2:a001479.

Mhlongo, M. I., Piater, L. A., Madala, N. E., Labuschagne, N., and Dubery, I. A. 2018. The chemistry of plant-microbe interactions in the rhizosphere and the potential for metabolomics to reveal signaling related to defense priming and induced systemic resistance. Front. Plant Sci. 9:112.

Miersch, O., Neumerkel, J., Dippe, M., Stenzel, I., and Wasternack, C. 2008. Hydroxylated jasmonates are commonly occurring metabolites of jasmonic acid and contribute to a partial switch-off in jasmonate signaling. New Phytol. 177:114-127.

Monteiro, R. A., Balsanelli, E., Wassem, R., Marin, A. M., BrusamarelloSantos, L. C., Schmidt, M. A., Tadra-Sfeir, M. Z., Pankievicz, V. C., Cruz, L. M., and Chubatsu, L. S. 2012. Herbaspirillum-plant interactions: Microscopical, histological and molecular aspects. Plant Soil 356:175-196.

Müller, T., Oradu, S., Ifa, D. R., Cooks, R. G., and Kräutler, B. 2011. Direct plant tissue analysis and imprint imaging by desorption electrospray ionization mass spectrometry. Anal. Chem. 83:5754-5761.

Mus, F., Crook, M. B., Garcia, K., Garcia Costas, A., Geddes, B. A., Kouri, E. D., Paramasivan, P., Ryu, M.-H., Oldroyd, G. E. D., Poole, P. S., Udvardi, M. K., Voigt, C. A., Ané, J. M., and Peters, J. W. 2016 Symbiotic nitrogen fixation and the challenges to its extension to nonlegumes. Appl. Environ. Microbiol. 82:3698-3710.

Nemes, P., Barton, A. A., and Vertes, A. 2009. Three-dimensional imaging of metabolites in tissues under ambient conditions by lase ablation electrospray ionization mass spectrometry. Anal. Chem. 81: 6668-6675. 
Nemes, P., and Vertes, A. 2007. Laser ablation electrospray ionization for atmospheric pressure, in vivo, and imaging mass spectrometry. Anal. Chem. 79:8098-8106.

Oldroyd, G. E., and Downie, J. A. 2008. Coordinating nodule morphogenesis with rhizobial infection in legumes. Annu. Rev. Plant Biol. 59:519-546.

Oldroyd, G. E., Murray, J. D., Poole, P. S., and Downie, J. A. 2011. The rules of engagement in the legume-rhizobial symbiosis. Annu. Rev. Genet. 45:119-144.

Pandey, S. S., Singh, S., Babu, C. S., Shanker, K., Srivastava, N. K., Shukla, A. K., and Kalra, A. 2016. Fungal endophytes of Catharanthus roseus enhance vindoline content by modulating structural and regulatory genes related to terpenoid indole alkaloid biosynthesis. Sci. Rep. 6:26583.

Pankievicz, V. C., Camilios-Neto, D., Bonato, P., Balsanelli, E., Tadra-Sfeir, M. Z., Faoro, H., Chubatsu, L. S., Donatti, L., Wajnberg, G., Passetti, F., Monteiro, R. A., Pedrosa, F. O., and Souza, E. M. 2016. RNA-seq transcriptional profiling of Herbaspirillum seropedicae colonizing wheat (Triticum aestivum) roots. Plant Mol. Biol. 90:589-603.

Pankievicz, V. C., do Amaral, F. P., Santos, K. F., Agtuca, B., Xu, Y., Schueller, M. J., Arisi, A. C. M., Steffens, M. B., de Souza, E. M., Pedrosa, F. O., Stacey, G., and Ferrieri, R. A. 2015. Robust biological nitrogen fixation in a model grass-bacterial association. Plant J. 81:907-919.

Pérez-Montaño, F., Alías-Villegas, C., Bellogín, R. A., del Cerro, P., Espuny, M. R., Jiménez-Guerrero, I., López-Baena, F. J., Ollero, F. J., and Cubo, T. 2014. Plant growth promotion in cereal and leguminous agricultural important plants: From microorganism capacities to crop production. Microbiol. Res. 169:325-336.

Phillips, D. V., Dougherty, D. E., and Smith, A. E. 1982. Cyclitols in soybean. J. Agric. Food Chem. 30:456-458.

Raun, W. R., and Johnson, G. V. 1999. Improving nitrogen use efficiency for cereal production. Agron. J. 91:357-363.

Reinhold-Hurek, B., and Hurek, T. 1998. Life in grasses: Diazotrophic endophytes. Trends Microbiol. 6:139-144.

Reinhold-Hurek, B., and Hurek, T. 2011. Living inside plants: Bacterial endophytes. Curr. Opin. Plant Biol. 14:435-443.

Richter, A., and Popp, M. 1992. The physiological importance of accumulation of cyclitols in Viscum album L. New Phytol. 121:431-438.

Roncato-Maccari, L. D. B., Ramos, H. J. O., Pedrosa, F. O., Alquini, Y., Chubatsu, L. S., Yates, M. G., Rigo, L. U., Steffens, M. B. R., and Souza, E. M. 2003. Endophytic Herbaspirillum seropedicae expresses nif genes in gramineous plants. FEMS Microbiol. Ecol. 45:39-47.

Sánchez-Martín, J., Heald, J., Kingston-Smith, A., Winters, A., Rubiales, D., Sanz, M., Mur, L. A., and Prats, E. 2015. A metabolomic study in oats (Avena sativa) highlights a drought tolerance mechanism based upon salicylate signalling pathways and the modulation of carbon, antioxidant and photo-oxidative metabolism. Plant Cell Environ. 38:1434-1452.

Sarkar, A., and Reinhold-Hurek, B. 2014. Transcriptional profiling of nitrogen fixation and the role of NifA in the diazotrophic endophyte Azoarcus sp. strain BH72. PLoS One 9:e86527.

Sessitsch, A., Hardoim, P., Döring, J., Weilharter, A., Krause, A., Woyke, T., Mitter, B., Hauberg-Lotte, L., Friedrich, F., Rahalkar, M., Hurek, T., Sarkar, A., Bodrossy, L., van Overbeek, L., Brar, D., van Elsas, J. D., and Reinhold-Hurek, B. 2012. Functional characteristics of an endophyte community colonizing rice roots as revealed by metagenomic analysis. Mol. Plant-Microbe Interact 25:28-36.

Shaw, L. J., Morris, P., and Hooker, J. E. 2006. Perception and modification of plant flavonoid signals by rhizosphere microorganisms. Environ. Microbiol. 8:1867-1880.

Shidore, T., Dinse, T., Öhrlein, J., Becker, A., and Reinhold-Hurek, B. 2012. Transcriptomic analysis of responses to exudates reveal genes required for rhizosphere competence of the endophyte Azoarcus sp. strain BH72. Environ. Microbiol. 14:2775-2787.

Smil, V. 2001. Synthetic fertilizers. Pages 128-131 in: Enriching the Earth: Fritz Haber, Carl Bosch and the transformation of world food production. MIT Press, Cambridge, MA, U.S.A.

Spaepen, S., and Vanderleyden, J. 2011. Auxin and plant-microbe interactions. CSH Perspect. Biol. 3:a001438.

St-Pierre, B., Vazquez-Flota, F. A., and De Luca, V. 1999. Multicellular compartmentation of Catharanthus roseus alkaloid biosynthesis predicts intercellular translocation of a pathway intermediate. Plant Cell 11: 887-900.

Stopka, S. A., Agtuca, B. J., Koppenaal, D. W., Paša-Tolić, L., Stacey, G., Vertes, A., and Anderton, C. R. 2017. Laser-ablation electrospray ionization mass spectrometry with ion mobility separation reveals metabolites in the symbiotic interactions of soybean roots and rhizobia. Plant J. 91:340-354.

Stopka, S. A., Samarah, L., Shaw, J. B., Liyu, A. V., Veličković, D., Agtuca, B. J., Kukolj, C., Koppenaal, D. W., Stacey, G., and Paša-Tolić, L. 2019.
Ambient metabolic profiling and imaging of biological samples with ultrahigh molecular resolution using laser ablation electrospray ionization 21 tesla FTICR mass spectrometry. Anal. Chem. 91: 5028-5035.

Streeter, J., Lohnes, D., and Fioritto, R. 2001. Patterns of pinitol accumulation in soybean plants and relationships to drought tolerance. Plant Cell Environ. 24:429-438.

Streeter, J. G. 1980. Carbohydrates in soybean nodules: II. Distribution of compounds in seedlings during the onset of nitrogen fixation. Plant Physiol. 66:471-476.

Streeter, J. G., and Bosler, M. E. 1976. Carbohydrates in soybean nodules: Identification of compounds and possible relationships to nitrogen fixation. Plant Sci. Lett. 7:321-329.

Subramanian, S., Stacey, G., and Yu, O. 2007. Distinct, crucial roles of flavonoids during legume nodulation. Trends Plant Sci. 12:282-285.

Sutton, M. A., Oenema, O., Erisman, J. W., Leip, A., van Grinsven, H., and Winiwarter, W. 2011. Too much of a good thing. Nature 472:159-161.

Tadra-Sfeir, M. Z., Souza, E. M., Faoro, H., Müller-Santos, M., Baura, V. A., Tuleski, T. R., Rigo, L. U., Yates, M. G., Wassem, R., Pedrosa, F. O., and Monteiro, R. A. 2011. Naringenin regulates expression of genes involved in cell wall synthesis in Herbaspirillum seropedicae. Appl. Environ. Microbiol. 77:2180-2183.

Tenenboim, H., and Brotman, Y. 2016. Omic relief for the biotically stressed: Metabolomics of plant biotic interactions. Trends Plant Sci. 21: 781-791.

Tilman, D., Balzer, C., Hill, J., and Befort, B. L. 2011. Global food demand and the sustainable intensification of agriculture. Proc. Natl. Acad. Sci. U.S.A. 108:20260-20264.

Tiwari, R., Awasthi, A., Mall, M., Shukla, A. K., Srinivas, K. S., Syamasundar, K., and Kalra, A. 2013. Bacterial endophyte-mediated enhancement of in planta content of key terpenoid indole alkaloids and growth parameters of Catharanthus roseus. Ind. Crops Prod. 43:306-310.

Vacheron, J., Desbrosses, G., Bouffaud, M.-L., Touraine, B., MoënneLoccoz, Y., Muller, D., Legendre, L., Wisniewski-Dyé, F., and PrigentCombaret, C. 2013. Plant growth-promoting rhizobacteria and root system functioning. Front. Plant Sci. 4:356.

van Der Heijden, R., Jacobs, D. I., Snoeijer, W., Hallard, D., and Verpoorte, R. 2004. The Catharanthus alkaloids: Pharmacognosy and biotechnology. Curr. Med. Chem. 11:607-628.

Van Moerkercke, A., Fabris, M., Pollier, J., Baart, G. J., Rombauts, S., Hasnain, G., Rischer, H., Memelink, J., Oksman-Caldentey, K.-M., and Goossens, A. 2013. CathaCyc, a metabolic pathway database built from Catharanthus roseus RNA-seq data. Plant Cell Physiol. 54:673-685.

Veličković, D., Agtuca, B. J., Stopka, S. A., Vertes, A., Koppenaal, D. W., Paša-Tolić, L., Stacey, G., and Anderton, C. R. 2018. Observed metabolic asymmetry within soybean root nodules reflects unexpected complexity in rhizobacteria-legume metabolite exchange. ISME J. 12:2335-2338.

Verma, P., Mathur, A. K., Srivastava, A., and Mathur, A. 2012. Emerging trends in research on spatial and temporal organization of terpenoid indole alkaloid pathway in Catharanthus roseus: A literature update. Protoplasma 249:255-268.

Wanek, W., and Richter, A. 1997. Biosynthesis and accumulation of Dononitol in Vigna umbellata in response to drought stress. Physiol. Plant. 101:416-424.

Wasternack, C. 2007. Jasmonates: An update on biosynthesis, signal transduction and action in plant stress response, growth and development. Ann. Bot. 100:681-697.

Wasternack, C., and Hause, B. 2013. Jasmonates: Biosynthesis, perception, signal transduction and action in plant stress response, growth and development. An update to the 2007 review in Annals of Botany. Ann. Bot. 111:1021-1058.

Webster, G., Jain, V., Davey, M., Gough, C., Vasse, J., Denarie, J., and Cocking, E. 1998. The flavonoid naringenin stimulates the intercellular colonization of wheat roots by Azorhizobium caulinodans. Plant Cell Environ. 21:373-383.

Westhoff, P. 2009. The economics of biological nitrogen fixation in the global economy. Pages 309-328 in: Nitrogen fixation in crop production. Agronomy monograph no. 52. D. W. Enerich and H. B. Krishnan, eds. American Society of Agronomy, Madison, WI, U.S.A.

Widemann, E., Miesch, L., Lugan, R., Holder, E., Heinrich, C., Aubert, Y., Miesch, M., Pinot, F., and Heitz, T. 2013. The amidohydrolases IAR3 and ILL6 contribute to jasmonoyl-isoleucine hormone turnover and generate 12-hydroxyjasmonic acid upon wounding in Arabidopsis leaves. J. Biol. Chem. 288:31701-31714.

Zivy, M., Wienkoop, S., Renaut, J., Pinheiro, C., Goulas, E., and Carpentier, S. 2015. The quest for tolerant varieties: The importance of integrating "omics" techniques to phenotyping. Front. Plant Sci. 6:448. 